\title{
Spontaneous Rupture of a Bicuspid Aortic Valve in a Middle-Aged Weightlifter
}

\author{
Stylianos A Karvounaris ${ }^{1}$, Evaggelos Sivitanidis², Petros P Mavrommatis ${ }^{3}$, Georgios S Papaetis ${ }^{4,5}$ \\ ${ }^{1}$ Department of Cardiology, Evangelismos Hospital, Paphos, Cyprus \\ ${ }^{2}$ Department of Cardiac and Thoracic Surgery, The Interbalkan Medical Center of Thessaloniki, Pylaia, Thessaloniki, Greece \\ ${ }^{3}$ Cardiac Care Center, Paphos, Cyprus \\ ${ }^{4}$ Internal Medicine and Diabetes Clinic, Paphos, Cyprus \\ ${ }^{5}$ CDA College, Paphos, Cyprus
}

Received: 20/12/2020

Accepted: 23/12/2020

Published: $25 / 01 / 2021$

How to cite this article: Karvounaris SA, Sivitanidis E, Mavrommatis PP, Papaetis GS. Spontaneous rupture of a bicuspid aortic valve in a middle-aged weightlifter. EJCRIM 2021;8: doi:10.12890/2021_002234.

Conflicts of Interests: The Authors declare that there are no competing interests.

This article is licensed under a Commons Attribution Non-Commercial 4.0 License

\section{ABSTRACT}

We describe a 58-year-old Caucasian male weightlifter who presented with acute shortness of breath after finishing his extensive exercise routine. Acute aortic valve regurgitation, due to spontaneous rupture of a bicuspid aortic valve, was diagnosed. Urgent surgical intervention was carried out, during which the bicuspid aortic valve was resected and replaced with an On-X bileaflet mechanical valve. The patient remains asymptomatic and is treated with warfarin, being in excellent physical condition 4 years after aortic valve replacement.

\section{LEARNING POINTS}

- Spontaneous rupture of a bicuspid aortic valve, after heavy weightlifting, is a very rare cause of acute aortic valve regurgitation.

- Echocardiography is of vital importance to distinguish the reason for this medical emergency from other possible causes.

- Prompt diagnosis and surgical treatment can achieve excellent long-term results.

\section{KEYWORDS}

Acute aortic valve regurgitation, spontaneous rupture, bicuspid aortic valve, weightlifting

\section{INTRODUCTION}

Acute aortic valve regurgitation is an uncommon medical emergency and is mostly caused by aortic root dissection, infectious endocarditis, sinus Valsalva aneurysm rupture, thoracic trauma or spontaneous rupture of a calcified or myxomatous aortic valve [1, 2]. We describe a 58-year-old Caucasian male weightlifter who presented with acute onset dyspnoea after finishing his intense workout program. Acute aortic valve regurgitation, due to spontaneous rupture of a bicuspid aortic valve (BAV), was diagnosed. Prompt diagnosis and surgical treatment achieved excellent long-term results.

\section{CASE DESCRIPTION}

A 58-year-old Caucasian male weightlifter, with an unremarkable medical history, presented to our clinic complaining of acute onset of dyspnoea after finishing his intense workout program in the gym. He had never smoked and he had not taken any medications or anabolic drugs in the past. The patient was afebrile and dyspnoeic with 22 breaths per minute. His blood pressure was 130/60 mmHg and the heart 
rate was regular at 110 beats/minute. The physical examination revealed an early grade II diastolic murmur along the left sternal border, as well as fine crepitant rales in both lower lung fields. Laboratory investigations were within normal limits including white blood count, liver and renal function indices, electrolytes, thyroid profile and inflammatory markers. Three sets of blood cultures were negative.

His electrocardiogram (ECG) showed sinus tachycardia and chest radiography showed signs of lung congestion. The transthoracic echocardiogram (TTE) suggested severe aortic valve regurgitation (pressure half-time $250 \mathrm{~ms}$ ) (Fig. 1A) due to possible rupture (Fig. 1B). Normal dimensions and contractility of the left ventricle (ejection fraction 65\%) with mild hypertrophy were found. The aortic root, ascending aorta, aortic arch and descending aorta were normal without any evidence of dissection. No obvious vegetations or any shunted flow communicating with other cardiac chambers were found. Transoesophageal echocardiography (TOE) confirmed rupture of the aortic valve (Fig. 1C). It also clarified that the valve was bicuspid without any obvious vegetations (Fig. 1D). The patient was treated with intravenous furosemide and was referred to the cardiothoracic surgical team. An urgent surgical intervention was carried out, during which the ruptured $B A V$ was resected and replaced with an On-X bileaflet mechanical prosthetic valve (Fig. 2A,B). Cultures of the valve tissue were negative and no calcifications were found. The patient experienced an uneventful postoperative period. He remains asymptomatic and is treated with warfarin. He is in excellent physical condition 4 years after his aortic valve replacement surgery.

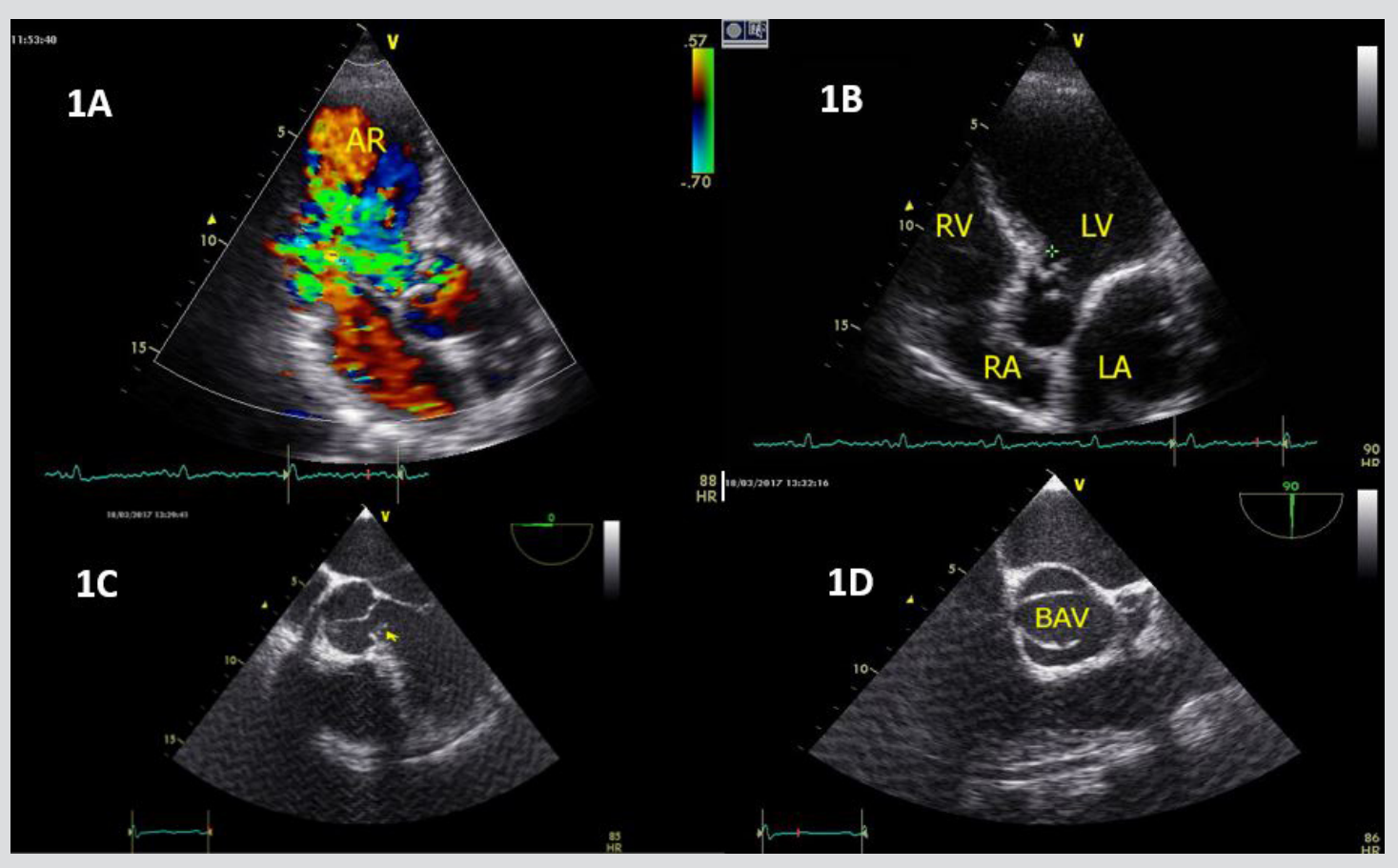

Figure 1. (A) TTE showing severe AV regurgitation. (B) TTE showing possible rupture of the AV (cross). (C) TOE showing possible rupture of the AV (yellow arrow). (D) TOE showing the BAV. AR: aortic regurgitation; $A V$ : aortic valve; BAV: bicuspid aortic valve; $L A$ : left atrium; LV: left ventricle; $R A$ : right atrium; $R V$ : right ventricle; TOE: transoesophageal echocardiography; TTE: transthoracic echocardiogram

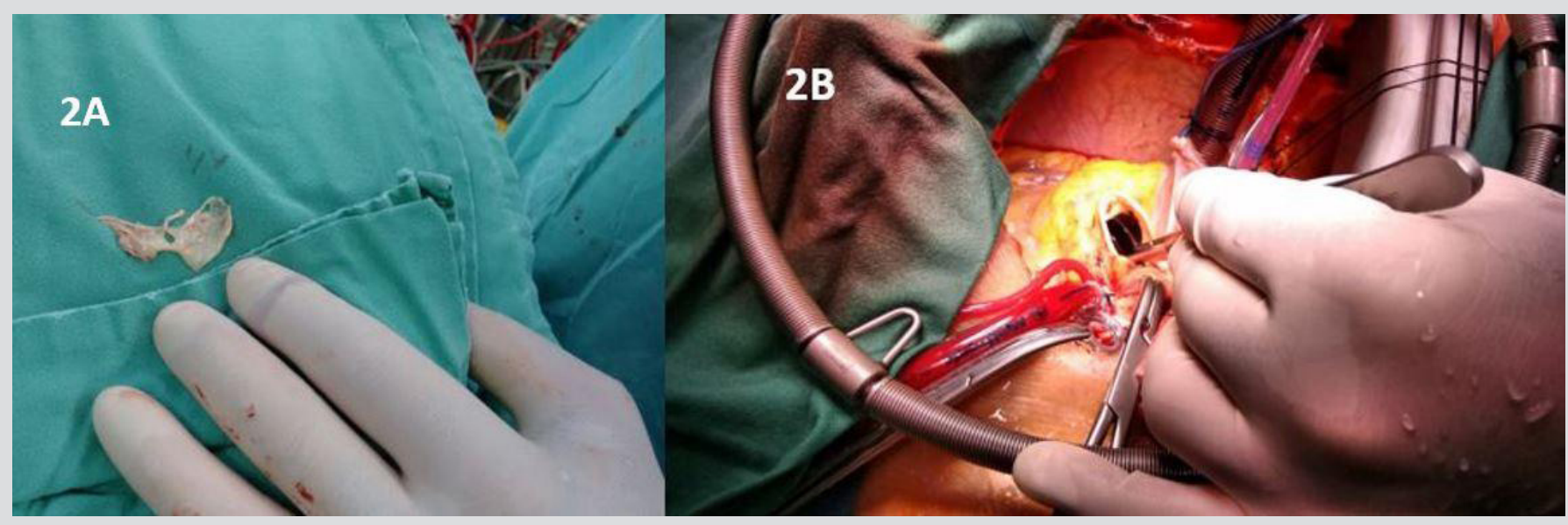

Figure 2. (A) The ruptured aortic valve (AV) is shown after resection. (B) Surgical removal of the AV 


\section{DISCUSSION}

Acute aortic valve regurgitation due to spontaneous rupture of a BAV is very rare. The first case report was published by Ciampricotti et al. in $1987^{[3]}$. To our knowledge, this is the first published report of spontaneous rupture of a BAV after heavy weightlifting. In fact, acute rupture as the first presentation of BAV is extremely rare. Our patient was completely asymptomatic before this event. The sudden onset of symptoms after his workout combined with the clinical, radiological and electrocardiographic findings support this association. There were also no signs of previous rheumatic or infectious endocarditis. He had not previously had a TTE, and had undergone only one routine ECG from his general practitioner, without any abnormal findings. The possibility of developing high blood pressure during his exhaustive training combined with the weakened aortic valve structure, could have led to the spontaneous rupture since no calcifications were found ${ }^{[3,4]}$. Interestingly, it was recently reported that heavy calcific deposits in BAV may extend across the orifice causing a perforation in the noncalcified portion of the opposing cusp ${ }^{[5]}$.

The diagnostic challenge for clinicians seeking to determine the exact cause of acute aortic regurgitation in patients with frank pulmonary oedema as in our patient, is difficult. Prompt TOE imaging evaluation is of vital importance in this critical setting, so as to establish the correct diagnosis and organize the appropriate treatment strategy. Proper surgical therapy can achieve an excellent long-term prognosis.

\section{REFERENCES}

1. Akinseye OA, Pathak A, Ibebuogu UN. Aortic valve regurgitation: a comprehensive review. Curr Probl Cardiol 2018;43:315-334.

2. Flint N, Wunderlich NC, Shmueli H, Ben-Zekry S, Siegel RJ, Beigel R. Aortic regurgitation. Curr Cardiol Rep 2019;21:65.

3. Ciampricotti R, el Gamal M, Mashhour YA. Acute aortic regurgitation due to spontaneous rupture of a bicuspid aortic valve: detection by echocardiography. Clin Cardiol 1987;10:484-486.

4. Moran SV, Casanegra P, Maturana G, Dubernet J. Spontaneous rupture of a fenestrated aortic valve. Surgical treatment. J Thorac Cardiovasc Surg 1977;73:716-718.

5. Roberts WC, Kale I, Roberts CS. Perforation of a stenotic congenitally bicuspid aortic valve cusp by heavy calcium in the other cusp. Am J Cardiol 2020;125:299-301. 